${ }^{1}$ Institute of Social and Preventive Medicine, University of Lausanne

2 Department of Social and Community Psychiatry, University Psychiatric Services Bern

3 Substance Abuse Unit, University of Lausanne

${ }^{4}$ Federal Office of Public Health, Bern

\title{
Appropriateness of methadone maintenance treatment for opiate addiction: evaluation by an expert panel
}

\section{Summary}

With some 30000 dependent persons, opiate addiction constitutes a major public health problem in Switzerland. The Swiss Federal Office of Public Health (FOPH) has long played a leading role in the prevention and treatment of opiate addiction and in research on effective means of containing the epidemic of opiate addiction and its consequences. Major milestones on that path have been the successive "Methadone reports" published by that Office and providing guidance on the care of opiate addiction with substitution treatment. In view of updating the recommendations for the appropriateness of substitution treatment for opiate addiction, in particular for the prescription of methadone, the FOPH commissioned a multi-component project involving the following elements.

- A survey of current attitudes and practices in Switzerland related to opiate substitution treatment

- Review of Swiss literature on methadone substitution treatment

- Review of international literature on methadone substitution treatment

- National Methadone Substitution Conference

- Multidisciplinary expert panel to evaluate the appropriateness of substitution treatment.

The present report documents the process and summarises the results of the latter element above.

The RAND appropriateness method (RAM) was used to distil from literature-based evidence and systematically formulated expert opinion, areas where consensus exist on the appropriateness (or inappropriateness) of methadone maintenance treatment (MMT) and areas where disagreement or uncertainty persist and which should be further pursued.
The major areas which were addressed by this report are

- Initial assessment of candidates for MMT

- Appropriate settings for initiation of MMT (general and special cases)

- Appropriateness of methadone supportive therapy

- Co-treatments and accompanying measures

- Dosage schedules and pharmacokinetic testing

- Withdrawal from MMT

- Miscellaneous questions

- Appropriateness of other (non-methadone) substitution treatment

Summary statements for each of the above categories are derived from the panel meeting and presented in the report.

In the "first round", agreement was observed for $31 \%$ of the 553 theoretical scenarios evaluated. The "second round" rating, following discussion of divergent ratings, resulted in a much higher agreement among panellists, reaching $53 \%$ of the 537 scenarios. Frank disagreement was encountered for $7 \%$ of all scenarios. Overall $49 \%$ of the clinical situations (scenarios) presented were considered appropriate. The areas where at least $50 \%$ of the situations were considered appropriate were "initial assessment of candidates for MMT", the "appropriate settings for initiation of MMT", the "appropriate settings for methadone supportive treatment" and "Appropriateness of other (non-methadone) substitution treatment". The area where there was the least consensus on appropriateness concerned "appropriateness of withdrawal from MMT" (6\%).

The report discusses the implications and limitations of the panel results and provides recommendations for the dissemination, application, and future use of the criteria for the appropriateness of MMT.

The RAND Appropriateness Method proved to be an accepted and appreciated method to assess the appropriateness of 
methadone maintenance treatment for opiate addicts. In the next step, the results of the expert panel process must now be combined with those of the Swiss and international literature reviews and the survey of current attitudes and practices in Switzerland, to be synthesized into formal practice guidelines. Such guidelines should be disseminated to all concerned, promoted, used and rigorously evaluated for compliance and outcome.

Keywords: Methadone - Quality of care - Appropriateness of care Substance abuse - Opiate - Guidelines

\section{Background and purpose}

\section{Importance of the problem and current issues ${ }^{1}$}

In Switzerland, opiate addiction constitutes a major public health problem. Some 30000 persons are dependent on hard drugs, mainly heroin. Such addiction carries with it increased risk of infection from HIV and hepatitis. Yearly, some 100 intravenous drug users die from AIDS and another 200 die from other causes, mainly drug overdose. In the countries of the European Union, some 1 to 1.5 million people are dependent on illicit opiates, mainly heroin. Addiction poses serious physical, psychological and social risks to those suffering from it and to others. One of the main aspects that induces these risks is the fact that, when people become more dependent on heroin, they need it more frequently and they need a larger dose. Their lives change into a constant quest for drugs and for money to buy them. Physical dependence becomes a way of life. One way of helping addicts reduce the unhealthy aspects of their addiction is substitution treatment, i.e., by prescribing a substitute drug for the illicit opiate. Since its introduction as a substitution drug in the 1960s methadone is the most popular substitution drug. It is estimated that $90 \%$ of opiate substitution treatment in European Union countries consists of methadone substitution treatment. In Switzerland some 18000 persons are treated with methadone.

The presumed advantages of substitution treatment are:

- more hygienic administration of drugs;

- care provider contact with the addict; the possibility for starting psychosocial treatments to handle other problems related to heroin addiction, such as psychosocial and legal problems, but also addiction to other drugs (e.g., cocaine);

- a possibility for the addict to stabilise his/her life, as substitution drugs are administered on a less frequent and more systematic basis;

- lower mortality.

\footnotetext{
1 Largely based on Van Beusekom and Iguchi (2001).
}

Although methadone is the most established drug for substitution treatment, it is still under continuous discussion. This discussion focuses, among other things, on adequate dosing, the efficacy of methadone as a substitution drug, the role of additional psychosocial treatments and the optimal duration of methadone maintenance treatment.

\section{The present project}

The Swiss Federal Office of Public Health (FOPH) has been a leading protagonist in the prevention and treatment of opiate addiction and in research on effective means of containing the epidemic of opiate addiction and its consequences. As milestones on that path are the successive "methadone reports" published by the FOPH since the 1980s. The project described in this paper is part of a major project of the Substance Abuse Section of the FOPH, aimed at updating recommendations for the appropriateness of substitution treatment for opiate addiction, in particular for the prescription of methadone. Since the last updated "Methadone report" in 1995, the nature of drug dependency has evolved, as have the system of health care and its handling of drug dependencies. It was felt that the updated recommendations needed to reflect those changes. Updating the recommendations for the appropriateness of substitution treatment for opiate addiction, in particular for the prescription of methadone, was framed into a multi-component project involving the following elements:

- A survey of current attitudes and practices in Switzerland related to opiate substitution treatment

- Review of Swiss literature on methadone substitution treatment

- Review of international literature on methadone substitution treatment

- National Methadone Substitution Conference

- Multidisciplinary expert panel to evaluate the appropriateness of substitution treatment

This report describes the last element and presents the results of a Swiss multidisciplinary - multi-professional panel aimed at evaluating the appropriateness of substitution treatment for opiate addiction for the various situations and patients - or clients - encompassing the most frequent situations encountered in Switzerland. Primarily, methadone maintenance treatment was examined. The panel method used, known as the RAND appropriateness method (Brook et al. 1986; Park et al. 1986; Brook 1994; Fitch et al. 2001), was chosen because it is aimed at detecting and improving agreement among the panellists, but not forcing consensus in case of conflicting evidence or disagreement. Panel participants were provided with the other components of the 
programme, in particular the reviews of the Swiss and international literature that provided a foundation for evidencebased recommendations by the panel concerning the appropriate use of methadone maintenance treatment in Switzerland.

The full reports concerning other parts of this project can be obtained from the Swiss Federal Office of Public Health [drog-int@bag.admin.ch] or accessed as Portable Document Files (PDF) from the internet site [http://www.nasuko2001.ch/ Background/Background/showVortragListe (accessed May 2003)]. However, it was felt important that readers have access to brief summaries of the other elements that were available to the panel of Swiss experts. Therefore, the following section summarises the first four components of the project that preceded the expert panel meeting.

\section{Survey of current attitudes and practices in Switzerland (Herrmann 2001)}

In December 2000 and March 2001, some 850 Institutions and persons were contacted and sent a standard questionnaire concerning current practice of methadone substitution treatment. 369 questionnaires from 25 cantons could be analysed, including 12 from cantonal delegates for drug abuse, 16 chief medical officers, 129 practicing physicians, 17 regional and cantonal hospitals, seven psychiatric clinics, 17 clinics specialised in care of drug dependency, 71 chemists, 73 counselling agencies and drop-in centres, and 27 in-patient centres. The results cover some 10310 persons, representing about $55 \%$ of all patients in methadone substitution programmes. The report described the different cantonal laws and ordinances guiding substitution treatment, as well as the perception and assessment of current practice from the point of view of care-givers (from different settings) and health authorities.

\section{Review of Swiss literature on methadone substitution treat- ment (Rehm et al. 2001)}

The review of Swiss literature since 1995 examined methadone maintenance treatment (MMT) in Switzerland, with particular interest for its effectiveness and its role in the overall picture of opiate dependency and how it is managed in different clinical settings: general practitioners, low threshold institutions, psychiatric clinics, acute-care hospitals, prisons. The review indicates that although the results of most studies favour methadone substitution treatment, they are generally plagued by problematic methodological pitfalls, in particular, lack of an adequate control group. The report describes how studies in Switzerland have defined the indication for MMT, which dosage schedules they use, insisting that studies which use higher doses appear to have better success rates than those with lower dosage schedules. In this context, it also emphasises the advances in pharmacokinetic testing to target individual substitution needs. The review examines what combinations of preventive and therapeutic regimens are being used in Switzerland as adjuncts to methadone substitution, as well as the problems arising from these regimens, in particular drug interactions. The report concludes with a recommendation for far more rigorous design and implementation of studies from Switzerland. It also calls from a re-examination of dosage schedules (in particular in relation to individual differences in methadone metabolism), consideration of the role of psychiatric comorbidities, better access to social services, a more solid foundation for the indications for substitution (and with which substance) as well as its distinction from abstinencetargeted therapy, and finally, for more high quality studies on effectiveness and cost effectiveness on the system level (rather than on individual components of the treatment of opiate dependents).

\section{Review of international literature on methadone substitution treatment (Van Beusekom \& Iguchi 2001)}

The international literature review looked at guidelines for methadone maintenance treatment from 17 different countries, examining such elements as criteria for admission into the programme; initial, maximal and allowable take-home doses; the use of injectable methadone and the healthcare personnel that administer the treatment. It further examines important advances that have been reported in the international (non-Swiss) literature in the last five years, in particular concerning the pharmacokinetics of methadone and the confirmation of the effectiveness of MMT that has been demonstrated in several studies, in particular studies indicating the effectiveness of higher doses of methadone than have been reported in previous studies. Individual chapters are devoted to needs of special populations: people with HIV infection or tuberculosis, polydrug users and pregnant addicts; to psychosocial treatment and other motivational interventions; and to recommendations for guideline development, summarised according to the main phases of methadone treatment, as they were examined by the panel itself, that is, initiation, maintenance and cessation of MMT.

\section{National Methadone Substitution Conference (www.nasuko2001.ch)}

To inform a broad range of protagonists in the treatment of opiate addiction, encourage participation from a wide range of stake-holders and foster buy-in for future recommendations on substitution treatment, the FOPH convened a national conference in November 2001. In addition to the 
presentation of results of the other major phases of the overall project described above, workshops allowed participants to exchange information and experience on such themes as adapting treatment to motivational staging, methadone treatment in a residential setting, psychiatric co-morbidities, dosage schedules, multiple drug abuse (in particular cocaine, alcohol and benzodiazepine) and treatment objectives and planning.

\section{Intended readership and purpose}

The present report is intended for all those involved in the treatment of opiate dependent patients, to provide them with an overview of how a multidisciplinary expert panel, using a validated and standardised consensus decision process, considered various aspects of this important problem for today's society. Because of the detailed nature of the clinical questions examined, not all aspects and not all the nuances could be examined in the expert panel process. Therefore, in order to get a more complete picture of current state of the art treatment, the present report should be read in the context of the full reports concerning the other parts of this project and with reference to the detailed annexes. Supplementation with further commentary may be required before widely applicable practice guidelines can be formulated. The authors feel, however, that, in itself, the report constitutes a fundamental contribution to guiding future practice and improving the care of opiate dependent patients.

\section{Method}

To arrive at detailed criteria for the appropriateness of different aspects of methadone substitution treatment, the authors applied the RAND appropriateness method (RAM). This section presents an overview of the different steps involved in the RAM, as applied in this project. Briefly, based on an extensive literature review and the consultation of experts, a list of indications and a selection of treatment options for methadone maintenance treatment was prepared. Members of the expert panel were provided with this list of indications, the literature reviews and the other elements of the project. Following an initial and individual rating, the panel met to discuss the results of their first round of ratings. The indications were modified when needed and a second round of ratings took place during the panel meeting. The second round of ratings led to a list of indications for methadone maintenance treatment that were categorised as: appropriate, uncertain or inappropriate. These detailed indications can be used prospectively by a physician interested to examine the opinion of the panel expert for a particular patient case. A synthesis of the results has been prepared for this report.
The successive steps mentioned above are presented below in more detail.

\section{Literature review}

A detailed literature review was performed to synthesise the latest available scientific evidence on the methadone maintenance treatment, as indicated in the background section (Rehm et al. 2001; Van Beusekom \& Iguchi 2001).

\section{Compose list of indications and selection of treatment options}

Based upon the Swiss and international literature reviews, initial contact with experts and the results of the survey of current attitudes and practices in Switzerland, factors that contribute to the appropriateness of initiation, maintenance and cessation of substitution treatment for opiate addiction were identified during a 2-day retreat in March 2001. Further participants were two recognised national experts in substance abuse, two methodologists and a member of the FOPH. The above mentioned factors were expanded into an extended list of indications ("mini-scenarios" describing patient and setting characteristics) which differ from each other according to those factors. The factors that were taken into consideration had to be restricted in such a manner that a feasible number of indications (scenarios) was examined. Based on earlier experience, it was anticipated that the number of indications would be between 500 and 1500 in number.

Following the March meeting, the criteria were further refined, in response to on-going feedback from the experts in the panel. The scenarios were finally produced in the form of a matrix, which categorised opiate addicts or therapeutic situations in terms of various personal, environmental, and social characteristics (symptoms, past history, co-morbidities, etc).

\section{Situations evaluated}

The following Table (Tab. 1) shows the major categories of clinical situations (scenarios) that were evaluated by the panel. The numbering in front of each category will be used to present panel results in the "Results" section of this report and refers to the chapters of the detailed report of the full ratings of appropriateness that is available as an Annex (App. 4) to this paper.

\section{Table 1 Treatments evaluated}

1. Elements of initial assessment

2. Appropriate settings for initiation of MMT (general cases)

3. Appropriate settings for initiation of MMT (special cases)

4. Appropriate settings for methadone supportive therapy

5. Appropriateness of co-treatments and accompanying measures

6. Maximal dosages schedules and pharmacocinetic testing

7. Appropriateness of withdrawal from MMT

8. Miscellaneous questions

9. Appropriateness of other (non-methadone) substitution treatment 


\section{Definitions of treatments and terms}

Part of the preparation for the panel meeting and the panel meeting itself involved arriving at agreement on terms and definitions used in the descriptions of the clinical scenarios (indications) and the treatments to be evaluated. The definitions of each of the treatments, as well as the signs and symptoms used to create the clinical scenarios, as they were agreed upon by the panel, are included in Appendix 2, available at [http://www.bag.admin.ch/sucht/publikationen/ $\mathrm{d}$ /publikationen.htm\#TherapieHR]. For a discussion of the importance of the various treatments as well as the clinical factors making up the clinical scenarios (indications) the reader is referred to the two literature reviews, contained in separate documents (Rehm et al. 2001; Van Beusekom \& Iguchi 2001).

\section{Recruitment of experts}

A multidisciplinary panel of experts was recruited from relevant disciplines to participate in the assessment of the appropriateness of opiate substitution treatment for all clinical scenarios identified. Explicit efforts were made to find representation from among different disciplines - medical, paramedical and social, and to arrive at a mix of academia and grass roots practitioners, in-patient and out-patient care, and to include representatives of the three major language areas of Switzerland. For this panel, diversity of opinion was sought. Because the aim was to have panel composition represent the state-of-the-art level of thinking about substitution of opiate addiction in Switzerland, it was decided that all of the panellists would be Swiss residents.

15 persons were finally recruited to participate in the expert panel. An initial meeting of the panel of experts took place in June 2001 to introduce them to their tasks and to obtain input on the indication structure, definitions and literature reviews. For various reasons, after initial recruitment, four panel members were not able to participate in the entire rating process. The final composition of the expert panel is found in the acknowledgement section at the end of this report as well as in Appendix 1, available at [http://www.bag. admin.ch/sucht/publikationen/d/publikationen.htm\#TherapieHR].

\section{First round evaluation of appropriateness}

The literature review and the list of indications were provided to the experts who were asked to individually rate the appropriateness of using substitution treatment for each indication on a nine-point scale, ranging from extremely inappropriate $(=1)$ to extremely appropriate $(=9)$ for the patient described in the indication (see below). For each scenario, the panel members assessed the benefit-risk ratio for the "typical patient in Switzerland receiving care delivered by the typical care provider in the typical care-giver setting."

A total of 553 clinical scenarios, involving nine different categories, were evaluated in the first round by the expert panellists. On average the panellists invested about four hours evaluating the clinical scenarios (range 1.5-6 hours).

The two literature reviews prepared by the Institut für Suchtforschung (ISF) (Rehm et al. 2001) and RAND Europe (Van Beusekom \& Iguchi 2001) were generally considered complete and unbiased by all panellists. Panellists invested, on average, about nine hours to the study of the literature reviews (range 3-16 hours). They considered the reviews to be generally informative and indicated that they played a role in their evaluation of the appropriateness of various treatments in the first-round rating.

\section{Intermediate discussion with experts}

Following the receipt of this first round of ratings, individual discussions were conducted with the panellists to answer questions from the first round experience and prepare suggested modifications to the scenarios and definitions, to be considered at the panel meeting. During these interviews it became evident that changes were needed to the clinical scenarios in order to make them more clinically relevant.

\section{National Substitution Conference (NaSuKo)}

As a modification of the traditional approach to RAM panels, a description of the process and summary results of the first round ratings were presented as part of a National Substitution Conference (NaSuKo). In particular, areas of clear agreement and disagreement were demonstrated so as to encourage comments from a wide range of stake-holders, foster buy-in for the areas of agreement among protagonists present at the conference and create awareness of disagreement where it exists.

\section{Appropriateness panel meeting}

The panellists then were convened in November 2001 for a two-day meeting under the leadership of two experienced moderators, one experienced in the appropriateness method, the other a leading expert in the field of treatment of substance abuse. The aim was to intensively review and discuss the scenarios. 11 panellists participated in the two rounds of rating.

Panellists were given feedback from the first round rating, showing their own ratings in relation to the distribution of other panellists' ratings for all scenarios. The discussion focused on the indications and treatments where there was disagreement among the panellists during the first round and 
panellists were given the opportunity to modify the original list of indications and/or definitions. The final number of scenarios evaluated at the meeting was 537. After discussing each major category of the list of indications, they re-rated each indication individually. These second round ratings were used to identify the panel's determination of the appropriateness or inappropriateness of substitution treatment for each indication (for the precise operational and statistical definition of "appropriateness" see below). The tworound process focused on detecting consensus among the panel members. No attempt was made to force the panel to consensus.

\section{Analysis of panel results}

Following the panel meeting, the second round ratings were analysed, to distil areas of consensus concerning the appropriateness of substitution treatment, areas of consensus concerning the inappropriateness of such treatment and areas where no consensus exists and which might be considered as needing further rigorous evaluation.

\section{Defining appropriateness}

Following the standard procedure for the RAM, and using the scheme presented in Figure 1, each scenario was classified as "appropriate", "uncertain" or "inappropriate", based on the panel median rating $(1-3=$ inappropriate; $4-6=$ uncertain; 7-9 = appropriate) and the degree of intra-panel disagreement (i.e., all indications where there was disagreement were classified as "uncertain", irrespective of the panel median score). Disagreement was considered to be present when, at least three of the 11 ratings fell in the $1-3$ region and at least three in the 7-9 point region; agreement was present when no more than 3 ratings fell outside the 3-point category $(1-3 ; 4-6 ; 7-9)$ containing the median; all other cases formed an intermediate or indeterminate category.

The two elements of panel median rating and disagreement were combined to determine appropriateness categories of the different treatments for methadone substitution (For definition of disagreement, see text).

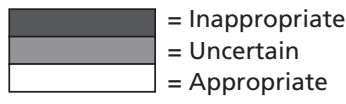

\section{Disagreement}

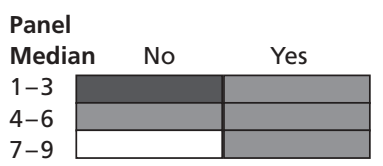

Figure 1 Determining appropriateness categories

\section{Results of panel}

\section{Agreement}

Summary results: The RAND appropriateness method does not force consensus. It is, however, particularly well designed to detect and quantify agreement (and disagreement) where and when it exists.

The first step in the process of analysis of the panel results was to look at the level of agreement among the panellists. Table 2 gives a summary of agreement for all 537 scenarios evaluated by the experts. It is presented by level of median rating of appropriateness and is organised in a similar fashion to the scheme for defining appropriateness in Figure 1 of the previous section.

Effect of multi-disciplinary discussion on agreement: An actual comparison between the first round rating, and the second round rating of appropriateness is difficult to perform because the content of the scenarios rated is not exactly the same between the two rounds. However, the impact of the panellists' interaction with other specialists can at least partially be analysed by looking at the differential agreement between the two rounds: the first round rating was done at home without any interaction and the second round was done following extensive discussion of the scenarios, the definitions, and the appropriateness of the different scenarios. In the first round, agreement was found for $31 \%$ of the 553 theoretical scenarios. The second round rating, following discussion of divergent ratings, resulted in a much higher agreement among panellists, reaching $53 \%$ of the 537 scenarios.

Further discussion of agreement can be found in the next section with reference to the appropriateness of different treatment scenarios. Complete analyses of agreement and disagreement can be found in the statistical Annex (App. 5), available at [http://www.bag.admin.ch/sucht/publikationen/ d/publikationen.htm\#TherapieHR].

\section{Appropriateness}

The full ratings of the appropriateness of various aspects of methadone substitution treatment (537 scenarios) are

Table 2 Median and disagreement categories (all 537 clinical scenarios)

\begin{tabular}{lrrr}
\hline \multirow{2}{*}{ Median } & \multicolumn{2}{l}{ Disagreement } & \multirow{2}{*}{ Total rows } \\
\cline { 2 - 3 } & No & Yes & \\
\hline $1-3$ & 140 & 5 & 145 \\
$4-6$ & 99 & 24 & 123 \\
$7-9$ & 263 & 6 & 269 \\
Total columns & 502 & 35 & 537 \\
\hline
\end{tabular}

Soz.- Präventivmed. 48 Suppl 1 (2003) S1-S14

(c) Birkhäuser Verlag, Basel, 2003 


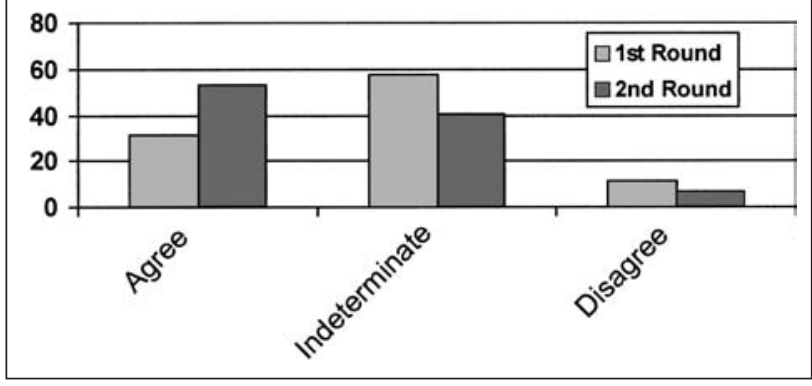

Figure 2 Percentage of agreement, 1st and 2nd rounds of rating

produced in Appendix 4, together with instructions for reading the ratings in Appendix 3. Both are available at [http://www. bag.admin.ch/sucht/publikationen/d/publikationen.htm\#TherapieHR].

Table 3 shows percentage of agreement and appropriateness categories for the major groupings of scenarios. As can be seen, the areas where there was clear-cut agreement for at least $50 \%$ of the scenarios were "elements of initial assessment", the "appropriate settings for initiation of MMT (general cases)", the "appropriate settings for methadone supportive treatment", and "appropriateness of co-treatments and accompanying measures".

Of particular interest is the $50 \%$ of the scenarios concerning withdrawal of MMT, where the panel results were neither appropriate nor inappropriate.

\section{Summary statements from evaluations of appropriateness}

One of the attractive aspects of the RAM is the ability to present clinical scenarios in sufficient detail that the experts, clinicians and other care-givers are able to envision the patient presented. This attention to clinical detail, however, carries with it the disadvantage that the results of the method (in the case of methadone maintenance treatment, a detailed evaluation of 537 scenarios) are hard to use, "as is", by the busy therapist. (Note: see the Recommendation section of the report for possibilities of addressing this difficulty through information technology.)

The following section presents a "digest" of the panel results for the 537 clinical scenarios evaluated by the expert panel. As a preliminary remark it is underscored that the statements below are often of a general nature and do not capture all the nuances and details discussed and rated by the panel. For accuracy, it is preferable to refer directly to the detailed results, which are included as Appendix 4 to this report, together with instructions on reading them (App. 3), both available at [http://www.bag.admin.ch/sucht/publikationen/d/publikationen.htm\#TherapieHR].

In addition, since exact definitions were agreed upon for all terms used in the clinical scenarios, it is also important to refer to them (App. 2, at the same site), as they may be somewhat different from the common usage of some of the terms.

\section{Initial assessment of candidates for MMT}

The following elements can be considered indicated (appropriate) for the initial assessment of patients for whom MMT is being considered. They are presented here in no specific order.

- Identity check

- HIV status

- Hepatitis serology + vaccination

- Consideration of psychiatric co-morbidities

- Consideration of social co-morbidities

- Duration and severity of dependency

- Ascertainment of poly-substance abuse or dependency

- Previous attempts at severance or substitution (number, results, mode)

Table 3 Percentage of agreement and appropriateness categories, by chapter

\begin{tabular}{|c|c|c|c|c|c|c|}
\hline Major category (no. of scenarios) & Disagree & Indeterminate & Agree & Inappropriate & Uncertain & Appropriate \\
\hline 1. Elements of initial assessment (16) & 0 & 13 & 88 & 0 & 0 & 100 \\
\hline 2. Appropriate settings for initiation of MMT & & & & & & \\
\hline (general cases) (264) & 1 & 35 & 64 & 22 & 24 & 54 \\
\hline $\begin{array}{l}\text { 3. Appropriate settings for initiation of MMT } \\
\text { (special cases) (33) }\end{array}$ & 12 & 46 & 42 & 24 & 24 & 52 \\
\hline $\begin{array}{l}\text { 4. Appropriate settings for methadone sup- } \\
\text { portive therapy ( } 21)\end{array}$ & 19 & 24 & 57 & 5 & 24 & 71 \\
\hline $\begin{array}{l}\text { 5. Appropriateness of co-treatments and accom- } \\
\text { panying measures (110) }\end{array}$ & 14 & 35 & 52 & 32 & 23 & 46 \\
\hline $\begin{array}{l}\text { 6. Maximal dosages schedules and pharma- } \\
\text { cokinetic testing ( } 32 \text { ) }\end{array}$ & 6 & 66 & 28 & 38 & 19 & 44 \\
\hline 7. Appropriateness of withdrawal from MMT (36) & 3 & 83 & 14 & 44 & 50 & 6 \\
\hline 8. Miscellaneous questions (19) & 32 & 42 & 26 & 47 & 42 & 11 \\
\hline $\begin{array}{l}\text { 9. Appropriateness of other (non-methadone) } \\
\text { substitution treatment (6) }\end{array}$ & 0 & 100 & 0 & 0 & 17 & 83 \\
\hline Total (537) & $7 \%$ & $41 \%$ & $53 \%$ & $26 \%$ & $25 \%$ & $49 \%$ \\
\hline
\end{tabular}

Soz.- Präventivmed. 48 Suppl 1 (2003) S1-S14

(c) Birkhäuser Verlag, Basel, 2003 
- Mode and frequency of opiate consumption

- Motivation

- Objectives of substitution treatment/contract

- Control (urinary or other) of unauthorised substance use

- Testing for tuberculosis (Mantoux)

- Personal and social situation/resources (including employment and living situation)

\section{Initiation of MMT: general guidelines}

The initiation of long-term MMT for opiate dependency was first examined in terms of general cases, considering the presence or absence of poly-substance abuse or dependency and the nature and severity of such poly-substance abuse.

The main question that the experts considered was: "Where is the appropriate place to treat the patient identified in the different clinical scenarios?" The clinical settings considered were:

- Primary or non-specialist care, without liaison to specialised resources,

- primary or non-specialist care, with liaison to specialised resources,

- specialised centre

Primary or non-specialist care, without liaison to specialised resources: The panel results indicate that it is appropriate to treat uncomplicated cases or those with predominantly somatic co-morbidities in this setting (i.e., patients without poly-substance abuse or dependency, without symptomatic psychiatric co-morbidities and without social co-morbidities), provided there is no poly-substance abuse or if such poly-substance abuse concerns alcohol, cannabis or sedatives and is not out of control.

In presence of symptomatic psychiatric co-morbidities, the panel results indicate that it is almost always inappropriate to initiate treatment in the primary care setting without liaison to specialised resources. Similarly the presence of polysubstance abuse that is out of control is a counter-indication to initiating MMT in this clinical setting.

Primary or non-specialist care, with liaison to specialised resources: The panel results indicate that the cases which are appropriate to treat in the absence of liaison to specialised resources (above) are at least equally as appropriate to treat with liaison to specialised resources. In addition, cases complicated by social or psychiatric co-morbidities and those involving poly-substance abuse with alcohol or cannabis or sedatives are appropriately treated in this setting.

The initiation of MMT was never clearly inappropriate for this setting, though there was considerable uncertainty for the more complicated cases.
Specialised centre: The initiation of MMT was generally appropriate for all cases, with the exception of uncomplicated cases without poly-substance abuse. For the more complicated cases, this was the only setting which was always clearly appropriate for the initiation of MMT.

\section{Initiation of MMT: specific cases}

Initiation of MMT during pregnancy: The panel results indicate that initiation of MMT in such cases is inappropriate, unless there is at least liaison to specialised resources. Where complications of pregnancy exist, it is preferable for treatment to be initiated only by a specialised centre.

Initiation of MMT for minors: For youth over 16, initiation of treatment can be done in the primary care setting with liaison to specialised resources. For persons under 16 it is only clearly appropriate to initiate MMT in a specialised centre. For the case of persons under 14, the panel felt it was clearly inappropriate to initiate such treatment other than in the specialised centre and even there the panel was clearly in disagreement about appropriateness of initiating MMT in such cases (five members voted 1 , five members voted 9 , one member voted 5).

Risk professions: For people working in professions where other people's lives depend on them, the panel disagreed about the initiation of MMT without recourse to specialised resources, but felt it was appropriate to do so in the settings where such resources were available, either on-site or in liaison.

Incarceration of known heroin addict: It would be appropriate for any of the considered settings to initiate MMT for such patients.

\section{Initiation of supportive methadone treatment: (special/emergency situations)}

Emergency hospitalisation of an opiate dependant person: The panel results indicate that it is appropriate to initiate supportive methadone treatment by any of the proposed levels of care in case of emergency hospitalisation of an opiate dependant person.

Run-away minor: There was uncertainty about the appropriateness of such treatment for run-away minors at the level of primary or non-specialist care, without liaison to specialised resources. On the other hand such treatment could be appropriately initiated by primary or non-specialist care, with liaison to specialised resources or in specialised centres. Release from incarceration of known drug misuser: There was disagreement about the appropriateness of such treatment for known drug misusers upon release from incarceration being undertaken by primary or non-specialist care, without liaison to specialised resources. On the other 
hand such treatment could be appropriately initiated by primary or non-specialist care, with liaison to specialised resources or in specialised centres.

Minors: The initiation of (emergency) methadone treatment for youth less than 18 years old was not considered appropriate in the setting of primary or non-specialist care, without liaison to specialised resources. In the special case of youth less than 14 years old, the panel results indicate that it is only appropriate to initiate such care in a specialised centre.

\section{Co-treatments for long-term MMT or accompanying mea-} sures (in addition to standard non-pharmacological support) This chapter examined the appropriateness of various cotreatments as a function of the co-morbidity profile of the patient as well as the phase of treatment.

Reinforced psychiatric treatment: The panel results indicate that, in the absence of symptomatic psychiatric co-morbidities, it is inappropriate to prescribe reinforced psychiatric treatment for most of the phases of treatment examined. There was, however, disagreement about situations of crisis or in presence of social co-morbidities during the withdrawal phase. On the other hand, in the presence of symptomatic psychiatric co-morbidities, it is always appropriate to prescribe reinforced psychiatric treatment for all of the phases of treatment examined. This was the area of the panel results where there was the greatest consensus.

Reinforced social support: The panel results indicate that, in the absence of social co-morbidities, it is inappropriate (or, at best uncertain) to prescribe reinforced social support for most of the phases of treatment examined. The major exception to this was during a crisis in the presence of concomitant psychiatric co-morbidities. On the other hand, in the presence of social co-morbidities, it is always appropriate to prescribe reinforced social support for all of the phases of treatment examined.

Other measures: The panel results indicate urinary control of unauthorised substance use (either for motivational reasons or for forensic reasons) can be considered appropriate, throughout most phases of treatment, with the exception of during the post-cure phase when there was uncertainty about the appropriateness of such measures. There was also disagreement among the experts concerning forensic control during the long-term maintenance phase. Other control measures were either of uncertain appropriateness (saliva analysis) or clearly inappropriate (hair and/or blood analysis).

The panel felt that methadone distribution via the pharmacy was clearly appropriate during the maintenance phase, but either inappropriate (initial or crisis phase) or questionable (withdrawal phase) during other phases (see also related item in chapter 8 , below).

\section{Dosage schedules and pharmacokinetic testing}

Results of the panel indicate that appropriate treatment generally involves an initial daily dosage of $20-30 \mathrm{mg} / \mathrm{day}$, to be incremented in a rapid phase by $5-10 \mathrm{mg} /$ day during 5-10 days. Following this, a slower incremental increase of $5-15 \mathrm{mg} /$ week may be appropriate, the aim being to achieve disappearance of withdrawal signs or adequate methadonemia levels. Because of varying addiction severity and inherent variations in methadone metabolism will be encountered, a certain amount of flexibility is required here.

The panel disagreed about whether the "appearance of signs of excessive dosage" was an appropriate criterion for attaining maintenance level dosage. However, the panel clearly considered that it was medically inappropriate to set a maximum on the daily dosage.

The panel indicated that it is inappropriate to apply pharmacokinetic testing to all patients. Appropriate indications would be when there are doubts about the adequacy of increasing dosage or before introducing a split dosage schedule. The panel results showed uncertainty about the appropriateness of general testing for all patients at or above $120 \mathrm{mg} / \mathrm{d}$. On the other hand, this latter situation was considered an appropriate indication for simple methadonemia.

\section{Appropriate indications for cessation of MMT}

This chapter examined the appropriateness of indications for terminating MMT, considering different scenarios related to the length of time since problems with unauthorized substances, the social and personal situation of the patient and the existence of credible projects for mid-term post-cure follow-up.

- The only clearly appropriate indication for terminating treatment, according to the panel results, would be when both patient and therapist agree to the termination, when the patient has had no problems with unauthorised substances for at least six months, when social and personal (including health) situation of the patient has improved and stabilised and treatment objectives have been attained.

- Conversely, the panel indicated that it would be inappropriate to terminate MMT if the social and personal (including health) situation of the patient has not improved and treatment objectives have not been attained.

- All other intermediate situations are either inappropriate or uncertain, with inappropriateness increasing as the patient situation resembles more the second situation above. 


\section{Miscellaneous questions related to MMT}

Maximum allowable daily take-home doses: The panel results suggest that six days would be the maximum allowable take home dose. Extremes of proposed alternatives (i.e., two or 14 days) were considered inappropriate.

Maximum allowable weekly holiday doses: The panel results suggest the quantity of methadone necessary for three weeks of treatment would be the maximum allowable holiday dose. Proposals for shorter periods manifested considerable disagreement among the experts. Setting the maximum limit of one gram of methadone (however long that would last) was considered inappropriate. The discussion about this point centred around the fact that some people require much higher doses and that if three weeks is considered the maximum allowable holiday dosage to be provided, one gram might not go very far towards those three weeks. Minimum waiting time before transferring distribution to pharmacy: There was disagreement among panel members about such a minimum (see related result in chapter 5 above), however the proposal that received the highest median vote was the proposition that there should not be a minimum waiting time.

Counter indications to MMT: The panel results on these questions showed considerable disagreement. This may be related to the double negative aspect of the question: "Is it appropriate to consider [__ $]$ as a counter indication to MMT?". Though this potential confusion was specifically pointed out during the panel meeting and before panellists voted on the appropriateness of the indications related to this, it cannot be excluded that confusion may have prevailed.

\section{Non-methadone substitution for opiate dependency}

It should be noted here that non-methadone substitution was not treated in sufficient detail to allow the development of precise guidelines for these alternative treatments to opiate dependency.

Bruprenorphine: The panel identified the following indications as appropriate for bruprenorphine:

- Hesitation between initiation of MMT or target of short term abstinence

- Treatment of methadone withdrawal syndrome

- Gastrointestinal intolerance to methadone

The panel was undecided about the appropriateness of bruprenorphine in younger $(<20$ years old) patients.

Heroin: The panel results indicate that "failure of MMT" is an appropriate indication for heroin substitution.

Naltrexone: The panel results indicate that it is appropriate to consider naltrexone for assistance in attaining abstinence.

\section{Panellists' assessment and acceptance of the method}

To evaluate the experts' opinion concerning the application of the RAM to the specific question of methadone maintenance treatment, they were asked to complete an evaluation questionnaire following the panel session. On a scale ranging from 1 (low) to 5 (high), the panellists estimated the validity and the utility of the method at a mean score of 4.1 and 4.4 respectively. The quality of the discussion was evaluated at a mean of 4.5 and the experts' own satisfaction in participating as a member of the panel was rated at 4.4.

The full evaluation of the expert panel is included in Appendix 6, available at [http://www.bag.admin.ch/sucht/publikationen/d/publikationen.htm\#TherapieHR].

\section{Discussion}

The RAND appropriateness method proved to be an accepted and appreciated method to assess the appropriateness of methadone maintenance treatment for opiate addicts. Experts generated new insights into the treatment of opiate addiction, in particular concerning areas where consensus exists, where it is absent and where uncertainty prevails. These insights can be considered as based on both the clinical expertise of the panellists and information from the scientific literature. In examining the potential treatments for the condition under consideration, the RAM was able to identify situations when treatments are inappropriate (that is, the risks outweigh the benefits) or appropriate (that is, the benefits outweigh the risks) and where there is uncertainty or disagreement.

It would seem important to make some remarks with respect to the interpretation of the panel results and in explanation of the method itself.

\section{Why the RAM?}

In the world of health care, the determination of correct treatment should ideally be based upon randomised clinical trials, rigorously testing one alternative against another. Unfortunately, this is not possible for the vast majority of health care provided and the same is also true in the field of treatment for substance abuse, as is evident from the conclusions of the review of Swiss and international literature on the subject (Rehm et al. 2001; Van Beusekom \& Iguchi 2001). However, the absence of solid evidence on the effectiveness or appropriateness of treatment does not free the care-giver or the policy maker from the often difficult choice of deciding what treatments to give or not to give, to allow or not to allow, to reimburse or not to reimburse. The RAM is certainly among the most studied of all methods of guidelines development and has been described as "the most respected 
approach to defining appropriate care" (Naylor 1998). For these reasons and because it is a validated method of providing reliable recommendations of appropriate care, even in the absence of other solid scientific evidence, it was chosen for this project.

\section{Panel composition}

The appropriateness of MMT was evaluated by a multidisciplinary panel of well-known and recognised Swiss experts in the field of addiction therapy. Although the panel was selected with care, the composition of the panel may influence the results of the evaluation. However, previous studies in which different panels of diverse composition rated the appropriateness of a similar medical procedure have shown that the effect of panel composition on the panel results is limited (Fraser et al. 1994; Bernstein et al. 1993; Vader et al. 1997). We feel therefore that it is not unreasonable to assume that the panel results generally reflect the opinions of Swiss addiction experts, both their agreement and their disagreement. The panel itself felt that the process used was capable of leading to valid guidelines for the appropriate use of MMT.

\section{Theoretical patients}

The clinical scenarios described in the indication matrices are composed of relevant patient characteristics, but do not represent actual patients. The panel members thus rated the appropriateness of a specific treatment for a theoretical patient. In practice, even for the cases where there was complete agreement among the panel members, there might be reasons to deviate from the recommendation of the expert panel, as a patient might have specific characteristics which beg for a different treatment strategy than the one recommended by the panel. In addition, the weight of these theoretical scenarios must be put into perspective of the frequency of actual cases. It is here that the use of the criteria, either prospectively to improve the appropriateness of care for addicted patients, or retrospectively to evaluate care that has been provided, will be most indispensable in determining the proportion of care that is actually appropriate.

\section{Need for other sources of information}

The RAM provides insight into the appropriateness of specific treatment options for specific sets of patients, but does not generate information on the most appropriate ways of dealing with patients from a legal or societal perspective. Therefore, the results and recommendations of this study should be put into the wider perspective of the legal and societal framework in which they are to be applied. It would also be important to supplement them with information on the frequency of actual use of various treatment options and with information from the scientific literature. Similarly, cost of MMT and other treatment options have not been examined explicitly by the panel.

\section{Appropriateness and necessity}

The RAM as applied here looked at the appropriateness of methadone maintenance treatment. The definition of appropriateness implies that to be appropriate, the expected health benefits (of the treatment) must outweigh the potential risks by a sufficiently wide margin that the treatment is worth doing. The fact that a treatment is appropriate does not mean that it is necessary or crucial, i.e., that it must be proposed by the care-giver or provided by the health care system.

\section{Alternative treatments}

By the same token, the results presented here do not say anything (except for the few scenarios addressing specific questions) about the appropriateness of other, alternative treatments. In fact, two treatments may be equally appropriate or, even if a treatment has been rated by the panel as appropriate, it may also be equally appropriate to prescribe no treatment.

\section{An international perspective}

The originality of the RAND method makes it difficult to compare results stemming from the Swiss panel with recommendations and guidelines developed with other methods. However, the review of international literature and guidelines (Van Beusekom \& Iguchi 2001), should be considered as an integral part of the RAM. Generally, it can be said that previously developed guidelines are heterogeneous, going from a general framework as in the Euromethwork (Verster \& Buning 2000) to very detailed and extensive guidelines such as those developed in the UK (Department of Health 2000), and encompassing more restrictive prescriptions, in particular on dosage such as the US guidelines (NIH Consensus Development Panel 1997).

Obviously, the political culture is crucial to the formulation of guidelines and scientific evidence or expert advice does not always predominate. Nonetheless, taking into consideration methodological and cultural limitations, we can observe both convergence and divergence in comparison with other guidelines.

Elements of convergence concern the more general aspects: initiation of maintenance treatment, accompanying measures for complex cases and scenarios for which criteria for stopping treatment reached consensus. Recommended vacation doses and the proposals for substitution in prison as 
well as the general principles on substitution with other substances are further areas which echo guidelines found elsewhere.

Concerning dosage schedules, recommended practice varies greatly among the countries studied in the international literature review (Van Beusekom \& Iguchi 2001). Not many countries state a minimum starting dose and those that do, have a higher starting dose than the one recommended by the panel. For example: Belgium $30 \mathrm{mg} /$ day, UK 10 $40 \mathrm{mg} /$ day, US $40 \mathrm{mg} /$ day. Most guidelines agree with the panel that a maximum dose would not be appropriate, although, Denmark, Finland, Germany, the UK and the US do set maximum doses.

Elements of divergence concern aspects of substitution treatment which are peculiar to a country open to programmes with a low threshold access, such as Switzerland, even though such low threshold access includes rigorous entry criteria. As an example, we can mention the differential recommendations for urine analysis between "forensic" and "motivational" check.

Another specific aspect of the RAND method concerns the considerations on "level of care". Treatment environment is a novel way of approaching guidelines that is not frequently encountered in previously published studies or recommendations. Exceptions would be the French guidelines which allow buprenorphine prescription by the general practitioner (non-specialised without liaison) and limit methadone to specialised treatment centres, and the fact that methadone substitution is simply not available for the general practitioner in the USA. On the other hand, the recommendations developed here indicate what the primary care provider can or cannot do without reference to specialised resources, compared to what can be expected of him in a network arrangement with access to specialised resources.

In the international literature, no country has gone into as much detail as the 537 scenarios proposed in the RAND method used here. This is most notable for situation of psychiatric co-morbidity, poly-substance abuse or the situations of young minors.

\section{Recommendations}

Based on the results of the panel and the processes leading up to it, the following recommendations are put forth as steps that will lead to improved care for those suffering from opiate addiction.

Facilitated access to user-friendly appropriateness criteria The results of a RAM panel can be used in different ways, both retrospectively and prospectively. Retrospective use of the panel results includes comparing the results of the panel with actual patient data and determining to which extent those patients were treated appropriately, according to the expert panel. Such an approach may be important in evaluating global progress towards more appropriate care, but has the disadvantage, for the individual patient, that treatment assessed has already been implemented. Such assessments are also of little or no value to the care-giver whose treatment has been assessed, post hoc.

In addition, retrospective evaluation of the appropriateness of care often suffers greatly from absence of sufficient and valid information required to assess appropriateness of care.

1. Nonetheless, it is recommended that, to allow assessment of appropriateness of care, using the criteria developed in this project, a minimal information and appropriateness dataset be created, disseminated, promoted and used by all involved in methadone substitution treatment. Such a minimal dataset should include the elements and details used to elaborate the patient scenarios evaluated by the experts. Synergy should be assured with a parallel project (methadone statistics) to create just such a minimal data set for all patients being treated for opiate dependence.

2. It is recommended that, as a follow-up to the present project, systematic use of such a database be combined with systematic collection of a minimal outcome dataset to allow follow-up of patient outcome in relation to the proposed criteria for appropriate care. Of prime importance is the collection of complete and high-quality data on treatments, their appropriateness and patient outcomes. If this systematic follow-up for all patients is unfeasible, a more limited cohort study could be planned, providing the same standards of full, complete and high-quality data collection.

The results of the RAM panel can also be used prospectively to develop clinical practice guidelines. Clinical practice guidelines are "systematically developed statements to assist practitioner and patient decisions about appropriate health care, i.e., decision aids for best practice". Clinical guidelines can take different forms; they can be documented in written form, but they can also take the form of a computer algorithm or a web-based information system. In the latter cases, care-givers can enter the patient characteristics into the computer and check whether the expert panel rated the chosen treatment as appropriate, uncertain, or inappropriate for a specific patient. In the present case with the multitude of scenarios the panel evaluated for methadone substitution treatment, an electronic version of the appropriateness criteria would be particularly well suited to the needs of having them readily available for consultation. 
The advantage of the prospective use of appropriateness criteria is that, 1) it helps the care-giver and patient make a decision on the appropriate treatment before that treatment is undertaken, rather than after the fact, and, 2) it promotes the documentation in the medical record of information related to the appropriateness of care, in case retrospective evaluation is later envisioned or applied.

3. It is therefore recommended that, as a follow-up to the present project, clinical guidelines in electronic or paper form be made generally available to both care-givers and patients to assist in the prospective choice of appropriate treatment, bearing in mind that clinical guidelines are meant to support and not to replace the decision making process of caregivers and that there may well be valid reasons to deviate from treatment suggested by the clinical practice guidelines.

\section{Implementation of practice guidelines}

The mere development and distribution of clinical practice and treatment guidelines is not enough to guarantee their utilisation. Special interventions should be carried out to foster the actual utilisation of guidelines in practice. These may include, for instance: academic detailing (i.e., face-toface, or small group interventions with targeted physicians or other health care professionals), educational material, reminders, feedback, interventions by opinion leaders. Combinations of various effective interventions to implement guidelines, including presentation of the guidelines at various events have, in the past, been shown to be more effective than single interventions.

4. It is recommended that a guidelines implementation strategy and programme be developed, financed and pursued.

\section{Research priorities}

For many treatment options, there was significant agreement on the appropriateness of the option: the panel either agreed that a specific approach was appropriate or inappropriate. However, a fairly substantial percentage of the ratings were uncertain. In these situations, research projects should be conducted in order to determine which approaches lead to the most desirable outcomes. Nevertheless, as it is impossible to subsidise research on all these treatments at the same time, priorities need to be set on which topics should be studied first. The panel ratings indicating considerable disagreement or uncertainty might be used to help target research to clarify those controversial or uncertain issues. For example, the panel results suggest the question of when (and if) it is appropriate to begin withdrawal of MMT is fraught with uncertainty, with the panel results indi- cating that for $50 \%$ of the scenarios withdrawal was neither appropriate nor inappropriate. This might be an ideal subject for rigorous clinical trials.

5. It is recommended that all actors involved in treatment of substance abuse consider fostering and funding highquality clinical studies to gradually, but systematically, clarify the many outstanding questions related to the appropriate care of opiate addicts. The notion of "high quality, valid studies" is particularly important in this field where much research is of questionable quality and adds little to our knowledge base, as was emphasised in the conclusions of the two literature reviews.

\section{Updating panel results}

As new studies on the effectiveness of MMT appear in the scientific literature, it is very important that the results of the expert panel are updated when developments in clinical research give reason to do so. To do this, however, it would be necessary to set up a "literature watch" which would scan the literature on the topic and flag areas where new publications from the literature might suggest the need to reconsider the panel's assessment of appropriateness for one or the other of the treatment approaches. This is particularly important to insure that any guidelines derived from the present project are kept up-to-date and not allowed to become obsolete. If the latter case were to occur, the effect would be counterproductive, i.e., it would promote inappropriate care rather than appropriate care.

6. It is recommended that the Federal Office of Public Health consider an on-going programme, either internally or externally, to monitor the state of scientific literature and its possible impact on the conclusions and criteria stemming from this project.

7. It is recommended that, no later than 2006 , the criteria for the appropriateness of MMT be fully re-evaluated.

\section{Supplement appropriateness criteria with other evidence}

Since the RAM panel was one part of a larger overall project,

8. It is recommended that, in a synthetic report, results of the panel be compared and supplemented with information on the frequency of actual use of various treatment options and with information from the scientific literature.

\section{International collaboration}

Given the societal importance of opiate abuse, the amount of resources needed to develop and maintain state-ofthe-art guidelines and the need for exchanging experience with others, some of the recommendations presented here could best be advantageously developed in collaboration 
with other partners at the supranational level. It is, for instance, the case for monitoring the literature, as scientific evidence is not expected to vary considerably between countries, and for conducting research projects. Furthermore, in previous and ongoing projects, it has proven feasible to conduct the development and updating of appropriateness criteria at the international or European level.

9. It is therefore recommended that the efforts of Switzerland to keep its standards and policies of opiate substitution therapy up-to-date continue to be integrated into international efforts along the same lines and that such integration and collaboration be reinforced.

\section{Conclusions}

The RAND Appropriateness Method proved to be an accepted and appreciated method to assess the appropriateness of methadone maintenance treatment for opiate addicts. It represents the first step in the development of nationally accepted practice guidelines. Such guidelines should be disseminated to all concerned, promoted, used and rigorously evaluated for compliance and outcome.

\section{Acknowledgment}

The authors wish to express their deep appreciation to the members of the expert panel for their competent contribution to the success of this project: Jacques Besson, Claudio Bosia, Elke Brusa, Barbara Gugger, Christian Junet, Silvie Petitjean, Françoise Rey, Bernadette Roos, Christina Stäubli, Rudolf Stohler, Peter Wyss. The following experts, initially recruited, were unable to participate in the panel meeting: Edith Aebischer, Christophe Bolli, Ignazio Cassis, Theo Vögtli.

This project was funded by the Swiss Federal Office of Public Health, Contract no. 01.000116.

\section{Annexes}

The following annexes mentioned in the report are available for download at: [http://www.bag.admin.ch/sucht/publikationen/d/publikationen.htm\#TherapieHR]

1. List of members of panel

2. Definitions

3. Instructions for reading appropriateness ratings

4. Full ratings of appropriateness for all 537 clinical scenarios evaluated

5. Summary statistics

6. Panellists' evaluation of the process

\section{References}

Bernstein SJ, Kosecoff J, Gray D, Hampton JR, Brook RH (1993). The appropriateness of the use of cardiovascular procedures: British versus U.S. perspectives. Int J Technol Assess Health Care 9: 3-10.

Brook RH (1994). The RAND/UCLA appropriateness method. In: McCormick KA, Moore SR, Siegel RA, eds. Methodology perspectives. Rockville, MD: US-DHHS: 59-70.

Brook RH, Chassin MR, Fink A, Solomon DH, Kosecoff J, Park RE (1986). A method for the detailed assessment of the appropriateness of medical technologies. Int J Technol Assess Health Care 2: 53-63.

Department of Health (1999). Drug misuse and dependence: guidelines on clinical management. London: The Stationery Office.

Fitch K, Bernstein SJ, Aguilar MD, et al. (2001). The RAND/UCLA appropriateness method user's manual. Santa Monica, CA: RAND Health.
Fraser GM, Pilpel D, Kosecoff J, Brook RH (1994). Effect of panel composition on appropriateness ratings. Int J Qual Health Care 6: 251-5.

Herrmann H (2001). Pratiques actuelles du traitement de substitution par la méthadone en Suisse (2000). Berne: Office fédéral de la santé publique.

Naylor CD (1998). What is appropriate care? N Engl J Med 338: 1918-20.

NIH Consensus Development Panel (1998). Effective medical treatment of opiate addiction: NIH consensus statement 1997. JAMA 280: 1936-43.

Park RE, Fink A, Brook RH, et al. (1986). Physician ratings of appropriate indications for six medical and surgical procedures. Am J Public Health 76: 766-72.

Rehm J, Uchtenhagen A, Dombrowski D (2001) Metaanalyse schweizerischer Arbeiten zur Verwendung von Methadon als Substitutionsmittel bei der Behandlung von Opioidabhängigkeit. Zürich: Institut für Suchtforschung.
Vader JP, Burnand B, Froehlich F, et al. (1997). Appropriateness of Upper Gastrointestinal Endoscopy: Comparison of American and Swiss criteria. Int J Qual Health Care 9: 87-92.

Van Beusekom I, Iguchi MY (2001). A review of recent advances in knowledge about methadone maintenance treatment. Leiden: RAND Drug Policy Research Centre.

Verster A, Buning E (2000). Methadone guidelines. http://www.euromethwork.org

Address for correspondence

Dr. John-Paul Vader, MD, MPH, PD

University of Lausanne

Institute of Social and Preventive Medicine

Rue du Bugnon, 17

CH-1005 Lausanne

Tel.: +41 213147280

Fax: + 41213144954

e-mail: John-Paul.Vader@inst.hospvd.ch

Soz.- Präventivmed. 48 Suppl 1 (2003) S1-S14

(C) Birkhäuser Verlag, Basel, 2003 